\title{
Study of an External Passive Shock-absorbing Mechanism for Walking Robots
}

\author{
Anthony David \\ JRL AIST/CNRS \\ Jean-Rémy Chardonnet \\ LIRMM - CNRS \\ JRL AIST/CNRS
}

\author{
Abderrahmane Kheddar \\ LIRMM - CNRS \\ JRL AIST/CNRS
}

\author{
Kenji Kaneko \\ IS - AIST
}

Kazuhito Yokoi
IS - AIST
JRL AIST/CNRS

\begin{abstract}
This paper proposes a compliant sole as an external shock-absorbing mechanism and investigates its effect comparatively to an ankle-located joint-flexible mechanism. The proposed mechanism is mounted under the HRP-2 humanoid feet only using simulation. The comparative evaluation has been conducted for contact resulting from walking using the HRP2 embedded pattern-generator. The characteristics of the sole material, Young and Poisson coefficients, are set following an adhoc minimization of their influence on the vertical acceleration and lateral inclination. Preliminary results suggest that the solution proposed is worth to be considered further and to be developed for real application use.
\end{abstract}

\section{INTRODUCTION}

A walking gait is generated by successive interactions between the humanoid's feet and the environment. This interaction is made through discreet contact formation, contact holding and contact release. The robustness of the walking gait depends in part on the quality of these interactions. A fast displacement of the robot requires precise control of these interactions and involves the capabilities for the hardware to deal with high impact forces. Furthermore, the future use of humanoid robots will not be made only on flat floors with smooth lands, as it is the case nowadays. In such case, it is more difficult to model with accuracy the environment. This induces premature contacts with the environment which create impacts and then vibrations on the robot's structure. Indeed, these impacts can excite mechanical resonance frequencies of the robot. Consequently, the movement of the robot is more or less jerky. In order to have smooth movements and to protect the robot structure and its embedded mechatronics, the robot must be able to absorb these impacts. To deal with this problem two directions are undertaken.

The first approach consists in using internal passive mechanisms. For example, shock-absorbing mechanisms are implemented in the feet of the robots. Bruneau et. al proposes a foot combined from four rigid bodies linked by flexible-joints [1] and claimed the difficulty in using finite-element method for modeling these flexibilities. The feet are composed of four bodies, related by three rotary joints with torsion springdampers. Internal passive mechanisms have been used for almost all humanoid robots such as Asimo [2] and HRP-2 [3]. The impact absorption mechanism of Asimo is composed of rubber bushes inserted into a guide. It deforms elastically in the vertical direction upon a force being transmitted from the sole. The shock-absorbing mechanism of HRP-2 is composed of three rubber bushes with dampers and connects the end link of each ankle to the sole link. Since the material of the rubber bushes is elastic, the bushes absorb the impacts.

The second approach consists in using external passive mechanisms. For example, Yamaguchi et. al use multicomposite soles with a complex arrangement [4]. This mechanism allows also to detect the landing path surface and to absorb the impact impulses. External passive mechanisms can also be used to protect robots when falling. The UKEMI method [5] is based on the utilization of flexible materials fixed on different points of the robot HRP-2P. These flexible materials allow to absorb impact and, so, to protect the robot.

The objective of this work is to investigate a shockabsorbing system consisting in a compliant sole covering the rigid structure of each foot and interacting directly with the floor. Unlike the compliant elements described in [5], our compliant sole is not present only to absorb impacts but the robot must also be able to walk with it. Depending on its design, it can also be helpful for walking, as explained, for example, in [1] [6]. Furthermore, external shock-absorbing mechanisms that were previously introduced are not modeled analytically, whereas here we propose to integrate the analytical model of our sole in the simulator presented in [7] [8]. These investigations are made, in particular, for the robot HRP-2 but our method extends to other humanoid robots. In a first time a sole composed of one compliant material is studied. Unlike the compliant sole developed in [9], our compliant sole will interact directly with the environment (it is not a compliant link between two rigid plates) and does not have internal sensors, like potentiometers. The second step is to evaluate, in simulation, the capacities of this sole to absorb shocks during walking gaits. For this purpose, the results obtained are compared with the shock absorbing mechanism implemented on HRP-2 [10]. Note that having an external deformable sole has also other benefits such as better adhesive properties and adapting, through deformation, to the irregularities of the terrain. In fact the idea is to investigate an all-in-one solution through an external sole foot-cover.

The organization of this paper is as follows. In Section II, the analytical models used are recalled. Next, in Section III, a simulation study to determinate the characteristics of the compliant sole is presented. Section IV discusses a comparative study between two shock-absorbing mechanisms: the compliant soles and the compliant joints. 


\section{Modeling}

We considered a compliant sole consisting simply on a parallelepiped shape mounted under each HRP-2 foot. To study this solution, one needs to model correctly the deformation of the sole and to extend the dynamic model of the rigid articulated structure to take into account this deformation. These two models (rigid robot structure and the deformable sole) are discussed separately and they are then summed-up. The models are written in 3-dimensions. Cartesian coordinates $(\vec{x}, \vec{y}, \vec{z})$, with $\vec{z}$ being the opposite direction to the gravity, are used. The simulation's numerical integration time-step is $\delta t$.

\section{A. Analytical models for poly-articulated rigid bodies}

In order to compute impact impulses or contact forces when contacts occur during walking, a constraint-based method is used because it proves to be very robust and require few parameters to set independently from the integration numerical method compared to penalty-based method. Constraint-based method requires writing relative velocities between pairs of potential contacts and split each pair of contact into orthogonal (normal) and tangent spaces. At the orthogonal space the Signorini non penetration constraint is written in terms of a complementary condition between the normal velocity and impact impulse or contact force (depending whether the contact is new or holding). In the tangent space the Coulomb friction constraint acts as a constraint, see [7] for more details.

1) Contact force model: The robot moves according to the following well-established dynamic equation:

$$
M(q) \ddot{q}+C(q, \dot{q})+G(q)=\Gamma+J_{c}^{T}(q) F_{c}
$$

with $M(q)$ the inertia matrix of the robot; $C(q, \dot{q})$ the centrifugal and Coriolis forces vector; $G(q)$ the gravity forces vector; $\Gamma$ the joint torques vector (including zeros for non-actuated joints); $J_{c}(q)$ the Jacobian matrix; $F_{c}$ the contact forces vector; $q$ the configurations vector (including robot attitude: position and orientation of the body in space).

Because of the presence of friction and impacts, we consider projection of contact constraints in the velocity space, avoiding problems described in [11]. Let $n_{i}$ be the unit normal vector defined for each potential contact $i$. The perpendicular plan to the vector $n_{i}$ is defined by the couples of vectors $t_{i}=$ $\left(t_{1 i}, t_{2 i}\right)$. A plan/plan contact can be discretized into $m$ contact points.

Since the floor is static, relative operational acceleration vector $a$ of the contact points can be written using the configuration acceleration vector $\ddot{q}$ as:

$$
a=J_{c} \ddot{q}+\dot{J}_{c} \dot{q}
$$

Combining (1) and (2) gives the link between $a$ and the corresponding contact force vector $F_{c}$ :

$$
\begin{aligned}
a & =J_{c} M^{-1} J_{c}^{T} F_{c}+J_{c} M^{-1}[\Gamma-C-G]+\dot{J}_{c} \dot{q} \\
& =\Lambda^{-1} F_{c}+a_{\text {free }}
\end{aligned}
$$

with $\Lambda=\left(J_{c} M^{-1} J_{c}^{T}\right)^{-1}$ the inverse of the operational inertia matrix (also called the Delassus operator); $a_{\text {free }}$ the free accelerations vector of the contact points.
Finally, the velocity update is obtained by integrating (3) with an explicit Adams formula of order 1:

$$
\begin{aligned}
v^{t} & =v^{t-\delta t}+\left(\Lambda^{-1} F_{c}^{t}+a_{\text {free }}\right) \delta t \\
& =\Lambda^{-1} \delta t F_{c}^{t}+a_{\text {free }} \delta t+v^{t-\delta t}=W F_{c}^{t}+v_{\text {free }}
\end{aligned}
$$

with $W=\Lambda^{-1} \delta t ; v$ the velocities vector of the contact points; $v_{\text {free }}$ the free velocities vector of the contact points. The Coulomb friction model is used as an additional constraint, that is: $\left\|F_{c i_{t}}\right\| \leq \mu_{i} F_{c i_{n}}$.

2) Impact impulse model: The impacts are determined with an event-based approach and are therefore considered as instantaneous. This hypothesis of instantaneous impacts, involves an abrupt change at the velocity level and, consequently, a discontinuity at the acceleration level. As for the contact forces model, the velocity of the given impact point $i$, depends on whether the impact remains (or not) inside the friction cone, that is [12]:

$$
\begin{aligned}
& v_{i_{n}}^{+}=-\epsilon v_{i_{n}}^{-}=-\epsilon J_{c i_{n}} \dot{q}^{-} \\
& \left\|p_{c i_{t}}\right\|<\mu_{i} p_{c i_{n}} \quad \text { sticking } \\
& \left\|p_{c i_{t}}\right\|=\mu_{i} p_{c i_{n}} \frac{v_{i_{t}}^{+}}{\left\|v_{i_{t}}^{+}\right\|} \quad \text { sliding }
\end{aligned}
$$

with $v_{i_{n}}^{+}$the normal velocity of the impact point $i$ just after the impact; $v_{i_{n}}^{-}$the normal velocity of the impact point $i$ just before the impact; $\epsilon$ the coefficient of restitution; $p_{c i_{t}}$ the tangential impact impulse vector and $p_{c i_{n}}$ the normal impact impulse. Eq. (5) is completed, for each of the $m$ impact points, with the following equations linking the impact impulse vector $p_{c}$ to the velocity vector $v^{+}: p_{c}=\Lambda\left(v^{+}-J_{c} \dot{q}^{-}\right)$.

\section{B. Analytical model of compliant soles}

Now, we consider that contact's position or velocity are the sum contribution of two connected sub-systems: a rigid and a deformable parts. The position of any point on the sole is the sum of the rigid motion induced by the robot structure and the deforming motion induced by sole flexibility. For this, a Finite Element Model is used in order to model the deformation of the compliant soles and integrate them in the previous rigid body analytical model. This approach is based on [13].

1) Mesh and model of the compliance sole: The software GMesh $^{1}$ is used to design then mesh, with linear field tetrahedron, the sole in 3D. The sole is composed of one isotropic compliant material, Fig.1. A linear elasticity behavior is considered because of the allowed deformation range relatively to the sole's size. Subsequently, the nodes displacement vector $U$ is a linear function of the external applied nodes' forces vector $F$, that is: $K U=F$; here $K$ is the stiffness matrix of the sole which expresses analytically in terms of the Young's modulus $E$ and the Poisson's coefficient $\nu$.

In order to obtain the expression of the displacements vector $U$, the Dirichlet nodes (i.e. attachment nodes to the rigid structure which do not deform) must be identified. A reduced stiffness matrix $K^{r}$, which is invertible, and its reduced

\footnotetext{
${ }^{1}$ http://www.geuz.org/gmsh/
} 
displacements vector $U^{r}$ are obtained by simply deleting the lines and columns corresponding to the Dirichlet nodes.
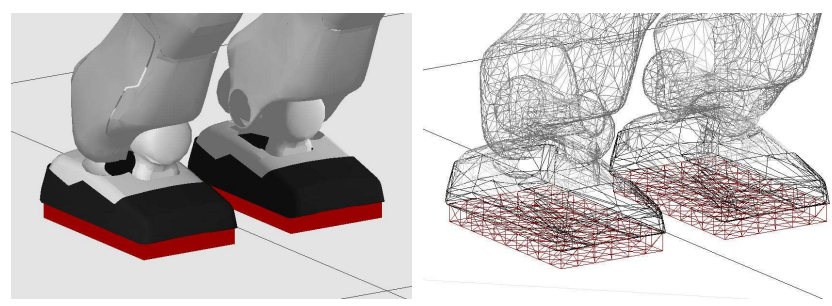

Fig. 1. The meshed sole mounted on the rigid part of each HRP-2 feet.

2) The complete contact force model: Here the rigid and deformable sub-systems are combined. This consists simply in adding the deformation velocities vector, $\dot{U}_{c}$, of the surface nodes that are contacting in eq. (4), such that:

$$
v^{t}=W F_{c}^{t}+v_{\text {free }}+\dot{U}_{c}^{t}
$$

$\dot{U}_{c}$ can be written as follows:

$$
\dot{U}_{c}^{t}=\frac{U_{c}^{t}-U_{c}^{t-d t}}{d t}=\frac{K_{c}^{-1} F_{c}^{t}-U_{c}^{t-d t}}{d t}
$$

with $K_{c}$ the stiffness matrix of the surface nodes in contact. The vector $U_{c}$ and the matrix $K_{c}$ are obtained using a classical condensation operation:

$$
\left[\begin{array}{ll}
K_{c c} & K_{c n} \\
K_{n c} & K_{n n}
\end{array}\right]\left[\begin{array}{c}
U_{c} \\
U_{n}
\end{array}\right]=\left[\begin{array}{c}
F_{c} \\
F_{n}
\end{array}\right]
$$

where the index $c$ denotes the nodes that are in contact and $n$ the remaining nodes.

In order to obtain a linear relation only between $U_{c}$ and $F_{c}$ but taking into account the influence of the other nodes, eq. (8) is developed:

$$
\begin{aligned}
& K_{c c} U_{c}+K_{c n} U_{n}=F_{c} \\
& K_{n c} U_{c}+K_{n n} U_{n}=F_{n}
\end{aligned}
$$

$U_{n}$ is then isolated in eq. (10) and its expression is introduced in eq. (9). As there is no external forces applied on internal nodes $\left(F_{n}=0\right)$, the expression of $F_{c}$ is:

$$
F_{c}=\left[K_{c c}-K_{c n} K_{n n}^{-1} K_{n c}\right] U_{c}=K_{c} U_{c}
$$

Finally, with eqs. (7) and (11), eq. (6) becomes:

$$
\begin{aligned}
v^{t} & =\underbrace{\left(W+\frac{K_{c}^{-1}}{d t}\right)}_{\widehat{W}} F_{c}^{t}+\underbrace{\left(v_{\text {free }}-\frac{U_{c}^{t-d t}}{d t}\right)}_{\hat{v}_{\text {free }}} \\
& =\widehat{W} F_{c}^{t}+\hat{v}_{\text {free }}
\end{aligned}
$$

This model is similar to the rigid bodies model given by eq. 4 .

When the robot is equipped with compliant soles, a specific model describing the impact is not needed since there is no abrupt change at the velocity level for the rigid part of the robot. The compliant soles gradually change the velocity profile of the robot along the normal $n$.

\section{Analytical model of compliant joints}

In order to evaluate the capability of this external passive mechanism, it is compared to the actual shock-absorbing mechanism of the HRP-2. This mechanism is mainly composed of an internal passive system [3]. Three absorber bushes with dampers connect the end link of the foot to the ankle, and an absorber rubber is placed between the sole rubber and the frame. Since this mechanism has a significant effect on the dynamic behavior of HRP-2, it must be modeled to be compensated during walking. Nakaoka et. al [10] use a virtual passive joint with three DoF to model this mechanism, see Fig. 2. It consists in one vertical translational axis, one pitch

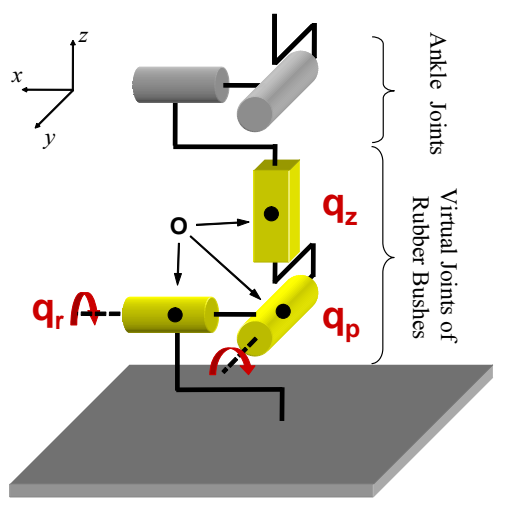

Fig. 2. The virtual passive joint model.

axis and one roll axis only. This compliant joint is located between the foot and the ankle, in the center of the three rubber bushes. A linear spring-damper force or torque model is used to simulate the deformation:

$$
\left\{\begin{array}{c}
f_{z}=-k_{\mathrm{TP}} q_{z}-k_{\mathrm{TD}} \dot{q}_{z} \\
\tau_{p}=-k_{\mathrm{RP}} q_{p}-k_{\mathrm{RD}} \dot{q}_{p} \\
\tau_{r}=-k_{\mathrm{RP}} q_{r}-k_{\mathrm{RD}} \dot{q}_{r}
\end{array}\right.
$$

with $q_{z}$ the translational value along the $z$-axis; $q_{p}$ and $q_{r}$ the angles of the pitch and roll axes respectively; $f_{z}, \tau_{p}$ and $\tau_{r}$ are the force and the torques given to the $z$, pitch and roll axes respectively; $k_{\mathrm{TP}}$ and $k_{\mathrm{RP}}$ are spring constants; $k_{\mathrm{TD}}$ and $k_{\mathrm{RD}}$ are damper constants. It is to note that these four constants were obtained by experimental tests on the real robot HRP-2, by identifying one axis at a time. Therefore, this model seems to fit well the real behavior of HRP-2. Finally, this compliant joint is included in the dynamic model of HRP-2, like the others joints.

HRP-2 has actually compliant soles (the black socks covering both feet). But these socks are thin, so their deformation is negligible, in particular relatively to the joint mechanism.

\section{Simulation StUdy}

The material used to design the compliant sole is characterized by three parameters: its thickness, the Young's modulus $E$ and the Poisson's coefficient $\nu$. In order to determinate these three parameters, ad-hoc simulation studies are realized. Two criteria are chosen: (i) the first criterion is the reduction of 
the acceleration peaks of the reference point along the gravity direction -it is along this direction that the effects of the impact are the most dominant, and (ii) the second criterion is the lateral inclination of the waist. These two criteria have antagonistic effects. Indeed, the more compliant the sole is, the more important acceleration's peaks reduction is -the velocity of the robot is damped progressively. However, the more compliant the sole is, the more important its deformation is; consequently, the more important the waist inclination is. This results very likely in highly unbalanced robot that will be hard to control. In view of all this discussion, it appears clearly that a compromise is to be found between these two criteria.

The thickness of the sole is taken to be $2 \mathrm{~cm}$ (max allowed, it can be optimized as well). The most important issue is to be sure that the maximum deformation never reaches the plastic domain in practical use, since in this case, the behavior switches to a rigid body.

For each of the two other parameters a range is chosen. For a perfect isotropic material, the theorytical value of the Poisson's coefficient is 0.25 . Furthermore, this coefficient is always inferior to 0.5 . If $\nu$ is equal to 0.5 , the material is perfectly incompressible. So, the following variation range is chosen: $\nu \in[0.01 \cdots 0.49]$. Concerning the Young's modulus, an elastic material, as gum or rubber for example, is desired. The gum has a Young modulus between 1.5MPa and 5.0MPa. So, the following range is chosen: $E \in[1 \cdots 10] \mathrm{MPa}$.

Two series of simulations are realized, one for each parameter. For the reduction of the acceleration peaks along the gravity direction, the robot is dropped above the ground from a height of $1 \mathrm{~cm}$. It is in standing position with straight legs. The maximum acceleration along the gravity direction is measured. For the lateral inclination, the robot starts with a standing position on the ground. It waist inclination is zero. Next, the waist inclination tends, gradually, toward a desired $14.3^{\circ}$. The difference between this desired inclination and the measured inclination is determined. This difference is due to the presence of the compliant sole.

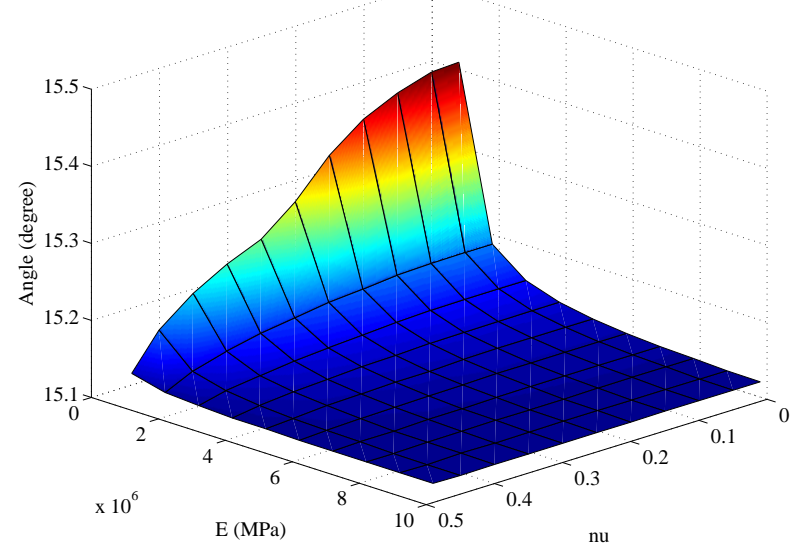

Fig. 3. Evolution of the waist inclination.

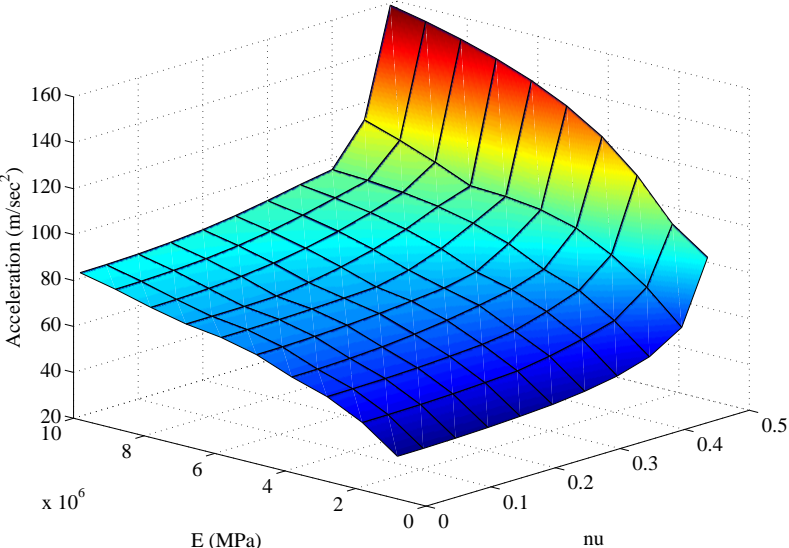

Fig. 4. Evolution of the maximum acceleration of the reference point along $z$.

Fig. 3 summarizes the results obtained for the inclination of the waist and Fig. 4 summarizes the results obtained for the maximum acceleration of the reference point along the gravity direction. The previous remark concerning the antagonistic effect of these criteria is visible.

Considering these results, the following parameters are chosen: $E=4.0 \mathrm{MPa}$ and $\nu=0.3$. These parameters give an acceptable inclination of the waist and a good decrease of the maximum acceleration of the reference point along the gravity direction.

\section{COMParative Study}

In order to measure the contribution of the proposed shock absorbing mechanism (the compliant sole), the results of three different simulations are compared. Firstly, HRP-2 is simulated without any shock absorbing mechanism, i.e. without any compliance. The results of this simulation are the reference to measure the contribution of each shock-absorbing mechanism. Secondly, HRP-2 is simulated with the real shock absorbing mechanism using the model recalled in section II-C. This corresponds to what is embedded on the real HRP-2. Thirdly, HRP-2 is simulated with the proposed shock absorbing mechanism, i.e. with the compliant soles. For each of the three simulations, the robot motion is made by the same reference control law. The reference trajectory is provided by the pattern generator described in [14]. The reference trajectory allows simulating a walking gait, where the robot avoids an obstacle in the spanning. This walking gait is composed of six steps and lasts six seconds. The maximum velocity of the reference point is equal to $0.3 \mathrm{~m} / \mathrm{sec}$, and the maximum length step is $0.45 \mathrm{~m}$. The reference trajectory is played directly with simple PD controls.

The evolution of a reference point, located on the waist of HRP-2, along the $z$ axis is compared. This axis corresponds to the opposite direction of gravity. Along this axis the effects of impacts, between the feet and the ground, and the effects of the compliant elements are the most visible. The evolution of 


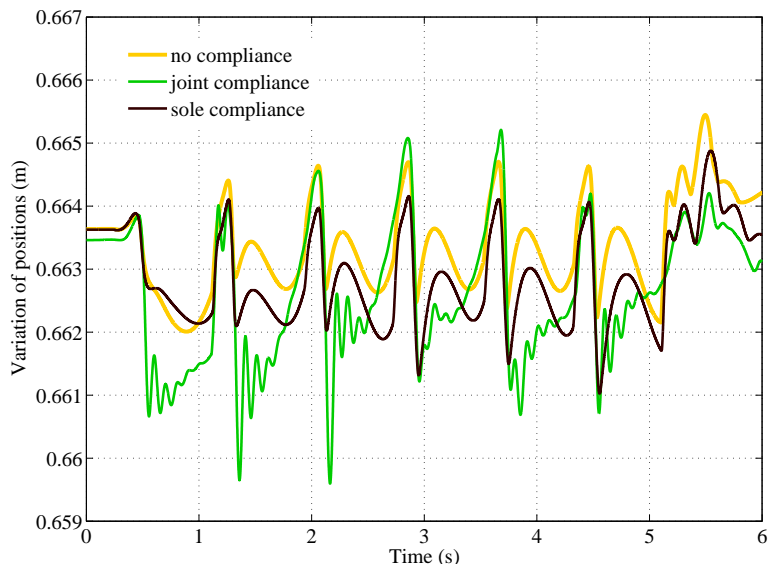

Fig. 5. Evolution of the reference point position along $z$. The actual position of the compliance sole need a $+2 \mathrm{~cm}$ shift; this is plotted without the shift for comparison purpose.

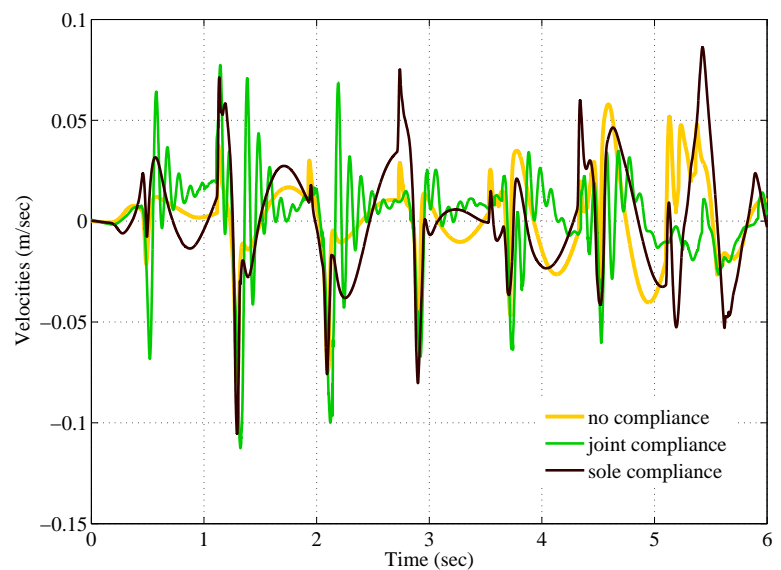

Fig. 6. Evolution of the reference point velocity along $z$.

the knee joint torque is also compared. The simulation results are summed up on Figs. 5 to 8 .

Fig. 5 shows the evolution of the position of the reference point along the $z$-axis. The position with the compliant soles is nearly similar to the position obtained without compliance. Indeed, after an impact, in the absence of external perturbations, the compliant sole behaves similarly as if it was globally a rigid body. The impact is absorbed with the deformation of the compliant sole. Then, the compliant sole keeps (globally) its new shape, thereby acting nearly as rigid, until the foot leaves the ground. The evolution of the position, in both cases, is not very important. On the opposite, the evolution of the position with the compliant joints is stronger. We can notice, unlike the two other curves, the presence of oscillations that are generated naturally by the joint spring-damper model, decreases fairly quickly due to the damper.

All these remarks are more contrasted on Figs. 6 and 7. Fig. 6 shows the evolution of the velocities of the reference

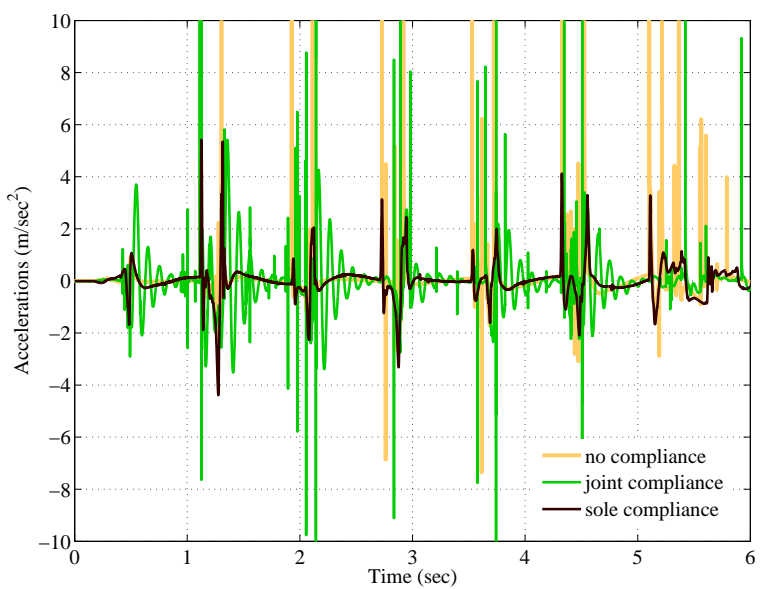

Fig. 7. Evolution of the reference point acceleration along $z$. The graph is cut between -10 and $10 \mathrm{~m} / \mathrm{sec}^{2}$ which can reach values of $60 \mathrm{~m} / \mathrm{sec}^{2}$ in the non compliant feet case, and up to $30 \mathrm{~m} / \mathrm{sec}^{2}$ with the joint compliance model.



Fig. 8. Evolution of the knee torque of the right leg.

point along $z$, and Fig. 7 shows the evolution of the acceleration of the reference point along $z$ as well. In particular, note the presence of strong oscillations of the velocity when compliant joints are used. Furthermore, we can see the presence of peaks (strong instantaneous perturbations) for the acceleration without compliance and with compliant joints. The presence of these peaks is due to impacts, when the feet enter in contact with the ground. The peaks are substantial (approximately $60 \mathrm{~m} / \mathrm{sec}^{2}$ ) without compliance because there is no mechanism to absorb shocks. The peaks are lower (approximately $30 \mathrm{~m} / \mathrm{sec}^{2}$ ) with compliant joints. The springdamper model, even if it does not eliminate completely these peaks, manages well to reduce them. On the other hand, peaks are absent when compliant soles are used. We can only see a moderate increase $\left(\approx 5 \mathrm{~m} / \mathrm{sec}^{2}\right)$ of the acceleration. This increase is not instantaneous. The compliant soles allow absorbing very well the peaks generated by the impacts.

Similar observations as before can be made with the evolu- 
tion of the joint torques. For example, that of the knee of the right leg (see Fig.8) have light oscillations when joint compliance is used. The two other curves do not exhibit these oscillations.

To sum-up, the absence of compliance involves the presence of strong instantaneous perturbations (peaks) when each foot enters in contact with the ground. Outside these phases of impacts, the behavior of HRP-2 is smoother. The presence of the compliant joints decreases the intensity of the peaks. However, these compliant joints induce oscillations and disturb the tracking control law. The resulting behavior of HRP2 is thus not completely smoother. Finally, the presence of compliant soles seems to solve both problems. The compliant soles remove the peaks without generating oscillations. The behavior is nearly continuous during impact phases and the tracking is good between two impacts.

Nevertheless, we must note that the four constants of the compliant joint were determined to represent the behavior of the real shock-absorbing mechanism that is actually mounted on HRP-2. The real system is not made of point flexible joints. More importantly, the parameters characterizing the actual shock absorbing mechanism of HRP-2 have not been optimized only for walking. They result from a compromise to satisfy other requirements, among which the constraints related to the design of the real mechanism (material used, integration, fabrication, etc.) which are limiting factors. Furthermore, the real shock-absorbing mechanism works also as a mechanical low-pass filter and respond to other issues that are not disclosed. Therefore, the comparison is certainly not fair; it should be seen only as a tendency.

\section{Conclusion and Perspective}

The presence of compliant soles allows absorbing the perturbations generated by the impacts. Furthermore, contrary to the compliant joint model, the compliant sole preserves a behavior close to the rigid non-compliant model when the contact holds. The control of the robot can consequently be realized with good precision, without being disturbed by any oscillations. However, a more complete study will be necessary in order to evaluate the capacity of the compliant sole to work as a mechanical low-pass filter, as the shock-absorbing mechanism of HRP-2.

Moreover the intrinsic design of the sole allows the compliance to be located on the external part of the foot, contrary to the shock-absorbing mechanisms that are located between two rigid parts of the robot (body structure and an external nearly rigid plate). Therefore, the compliant sole has an infinite number of DoF. It is not the case for internal mechanisms which have a finite and guided number of DoF. These infinite DoF offers good adhesive and adaptation to roughness capabilities in addition of a pressure distribution along all the compliant surface.

As explained in the introduction, this work is preliminary investigation prior to the development of a more optimized compliant sole for the humanoid robots. For example, a more refined shape could include an artificial toe joint only by tapering the front part of the sole. So, the next step will consist in improving the design for a new compliant sole having eventually more complex composition. In particular, it will be interesting to compose with more than one material in order to have a better absorption of impacts and a less residual deformation of the compliant sole with high adhesive and local deformation at the contacting surface.

\section{ACKNOWLEDGMENT}

This research was partially supported by Grant-in Aid for JSPS Fellows.

\section{REFERENCES}

[1] O. Bruneau, F. B. Ouezdou, and J.-G. Fontaine, "Dynamic walk of a bipedal robot having flexible feet," in IEEE/RSJ International Conference on Intelligent Robots and Systems, Maui, USA, October 2001, pp. 512-517.

[2] K. Hirai, M. Hirose, Y. Haikawa, and T. Takenaka, "The development of honda humanoid robot," in Proceedings of IEEE International Conference on Robotics and Automation, Leuven, Belgium, May 1998, pp. 1321-1326.

[3] K. Kaneko, F. Kanehiro, S. Kajita, H. Hirukawa, T. Kawasaki, M. Hirata, K. Akachi, and T. Isozumi, "Humanoid robot hrp-2," in Proceedings of IEEE International Conference on Robotics and Automation, New Orleans, USA, April 2004, pp. 1083-1090.

[4] J. Yamaguchi and A. Takanishi, "Multisensor foot mechanism with shock absorbing material for dynamic biped walking adapting to unknown uneven surfaces," in Proceedings of IEEE International Conference on Multisensor Fusion and Integration for Intelligent systems, Washington DC, USA, December 1996, pp. 233-240.

[5] K. Fujiwara, F. Kanehiro, S. Kajita, K. Kaneko, K. Yokoi, and H. Hirukawa, "Ukemi: Falling motion control to minimize damage to biped humanoid robot," in Proceedings of IEEE International Conference on Intelligent RObots and Systems, Lausanne, Switzerland, October 2002, pp. 2521-2526.

[6] S. Miyakoshi and G. Cheng, "Examing human walking characteristics with a telescopic compass-like biped walker model," in IEEE International Conference on Systems, Man and Cybernetics, Tokyo, Japan, October 2004, pp. 1538- 1543.

[7] J.-R. Chardonnet, S. Miossec, A. Kheddar, H. Arisumi, H. Hirukawa, F. Pierrot, and K. Yokoi, "Dynamic simulator for humanoids using constraint-based method with static friction," in Proceedings of IEEE International Conference on Robotics and Biomimetics, Kunming, China, December 2006, pp. 1366-1371.

[8] J.-R. Chardonnet, F. Keith, A. Kheddar, K. Yokoi, and F. Pierrot, "Interactive dynamic simulator for humanoid with haptic feedback," in Proceedings of the 17th CISM-IFToMM Symposium on Robot Design, Dynamics and Control, Tokyo, Japan, July 2008, pp. 317-324.

[9] J. Yamaguchi, A. Takanishi, and I. Kato, "Experimental development of a foot mechanism with shock absorbing material for acquisition of landing surface position information and stabilization of dynamic biped walking," in Proceedings of IEEE International Conference on Robotics and Automation, Nagoya, Japan, May 1995, pp. 2892-2899.

[10] S. Nakaoka, S. Hattori, F. Kanehiro, S. Kajita, and H. Hirukawa, "Constraint-based dynamics simulator for humanoid robots with shock absorbing mechanisms," in Proceedings of IEEE International Conference on Intelligent RObots and Systems, San Diego, USA, October 2007, pp. 3641-3647.

[11] D. Baraff, "Fast contact force computation for nonpenetrating rigid bodies," in SIGGRAPH, Orlando, USA, July 1994, pp. 23-34.

[12] W. J. Stronge, Impact Mechanics, C. U. Press, Ed. Press Syndicate of the University of Cambridge, 2000.

[13] C. Duriez, F. Dubois, A. Kheddar, and C. Andriot, "Realistic haptic rendering of interacting deformable objects in virtual environments," IEEE Transactions on Visualization and Computer Graphics, vol. 12, no. 1, pp. 36-47, January 2006.

[14] O. Stasse, B. Verrelst, P.-B. Wieber, B. Vanderborght, P. Evrard, A. Kheddar, and K. Yokoi, "Modular architecture for humanoid walking pattern prototyping and experiments," Advanced Robotics, Special Issue on Middleware for Robotics-Software and Hardware Module in Robotics System, vol. 22, no. 6, pp. 589-611, 2008. 